Results 168 men, median age 70 (range 34 -83), 50 women median age 61 (29-87), 28 treated for GI, 34 gynaecological \& 156 urological cancer were randomised to booklet $(n=68)$, nurse $(n=80)$ or gastroenterologist $(n=70) .30(44 \%)$ from the booklet and $4(5 \%)$ from the nurse arm crossed to the gastroenterologist. Groups were well balanced for baseline scores and patient characteristics. $66.5 \%$ of patients had a baseline IBDQ-B score indicating moderate/severe symptoms. Intention to treat analysis showed a mean improvement in IBDQ-B score in the booklet arm of 4.9 (95\% CIs 1.4-8.4), in the nurse arm 8.8 (6.9-11.2) and 10.3 (7.7-13.1) in the gastroenterologist arm. Improvement in IBDQ-B score was both clinically and statistically significant (compared to booklet) in the nurse $(p=0.04)$, gastroenterologist $(p=0.014)$ and combined treatment arms $(p=0.006)$. Outcomes in the nurse treated arm were not worse than those treated by the gastroenterologist $(p=0.428)$. Improvements were sustained over time.

Conclusion Targeted intervention following a detailed clinical algorithm can significantly ameliorate radiotherapy-induced GI symptoms. Most patients can be managed by a suitably trained and supported nurse. (Funding RfPB, NIHR)

Disclosure of Interest None Declared

\section{OC-069 CONSTITUTIVE ACTIVATION OF THE DNA DAMAGE RESPONSE PATHWAY IN CANCER REPRESENTS A DEREGULATED PATHWAY}

doi:10.1136/gutjnl-2013-304907.068

1.2, $\mathrm{S}$ Din, ${ }^{1} \mathrm{~T}$ Hupp. ${ }^{1} p 53$ Signal Transduction, Edinburgh Cancer Research UK Centre; ${ }^{2}$ GI Unit, Western General Hospital, Edinburgh, UK

Introduction The DNA damage response (DDR) is an innate cellular response allowing cells to halt the cell cycle and repair DNA damage sustained by activating various mechanisms. The efficacy of conventional cancer treatment modalities is related in their ability to induce DNA damage. Constitutive activation of the ataxia telangectasia mutated (ATM) dependent DDR and repair pathways have been reported in (pre) malignant human tissues and may undermine the efficacy of current cancer therapies. Inhibition of proteins involved in the DDR cascade is an attractive therapeutic concept that may overcome resistance to current cytotoxics and potentiate the effects of radiotherapy.

Methods A tumour microarray was created using 179 sporadic colorectal cancers; 152 were of the microsatellite stable phenotype. The microarray was interrogated using antibodies against proteins of the DDR signalling cascade. A colorectal cancer cell line model was utilised to asses the functionality of the constitutively activated DNA damage pathway. ATM inhibition in combination with ionising irradiation was analysed in the cell line model using radioactive quantification of DNA synthesis, flow cytometric cell separation, clonogenic survival and immunoblotting.

Results Phosphorylated Chk2 threonine-68, a surrogate marker of the DDR, was present in $22 \%$ of microsatellite-stable colorectal tumours and $33 \%$ of tumours with the microsatellite instability phenotype. High p53 staining was present in $53 \%$ of microsatellite stable cancers and $26 \%$ microsatellite instable cancers.

P21-null HCT116 cells display constitutive activation of the ATM DDR but display a defect in the ionising radiation induced S-phase checkpoint, termed radioresistant DNA synthesis. This radioresistant phenotype is associated with increased basal levels of Cdc25A protein, deficient DNA damage-induced degradation of Cdc25A and Chk2 mis-localisation. P21-null HCT116 and SW620 cells, which exhibit basal Chk2 threonine-68 phosphorylation, were unable to abrogate the S-phase checkpoint when treated with an ATM inhibitor, suggesting that the ATM-Chk2 arm is non-functional in these cells: inhibition of ATM did not potentiate the efficacy of ionising irradiation.
Conclusion In a colorectal cancer cell line model constitutive activation of the ATM DDR pathway reflected a non-functional pathway and inhibition of ATM in these circumstances was unable to potentiate the efficacy of ionising irradiation. Basal Chk2 threonine-68 phosphorylation in colorectal cancer may reflect a deregulated ATM DDR pathway and/or checkpoint adaption.

A predictive model is proposed that integrates functionality of the ATM-Chk2 axis, p53 mutation status and defects in DNA repair pathways when considering ATM inhibitor therapy.

Disclosure of Interest None Declared

\section{OC-070 PERCEIVED DELAY AMONG PATIENTS WITH COLORECTAL, STOMACH AND OESOPHAGEAL CANCER: ANALYSIS OF DATA FROM A NATIONAL GP AUDIT}

doi:10.1136/gutjnl-2013-304907.069

'C Dobson, 1," G Rubin. 'School of Medicine, Pharmacy and Health, Durham University, Stockton-on-Tees, UK

Introduction The UK has significantly poorer cancer survival rates than comparable countries and diagnostic delay is perceived to be a significant contributory factor to this. The RCGP National Audit of Cancer Diagnosis in Primary Care (2009/10) included data on 3655 patients with colorectal and gastro-oesophageal cancer, including free text comments on avoidable delays in diagnosis, as perceived by the participating GPs. The aim of this study was to identify the principal causes of delay, as perceived by GPs, and how they differ by cancer site. Methods Avoidable delay was reported for $36 \%$ of patients with colorectal cancer, $37 \%$ gastric cancer and $35 \%$ oesophageal cancer. Free text reports of the nature of the delay were available for 753 (28\%) colorectal, 87 (28\%) gastric and 164 (27\%) oesophageal cancer patients. An extended version of The Model of Pathways to Treatment (Walter et al 2011) was developed for use as the analytical framework. Comments were categorised by CD with uncertain cases discussed and resolved with GR. In order to validate GP perceptions of diagnostic delay we compared categorised primary care and referral intervals for patients with and without perceived delay. Results Primary care and referral intervals were significantly longer for patients with a perceived avoidable diagnostic delay ( $p=<0.0001$ ), for all three cancer sites. The commonest reasons for delay for colorectal, gastric and oesophageal cancer patients were GP appraisal (29\%, 14\%, 16\% respectively), referral delays (e.g. routine rather than 2 week wait) $(13 \%, 23 \%, 32 \%$ respectively) and investigation delays $(28 \%, 34 \%, 27 \%$ respectively). For colorectal cancer patients, help seeking delay was also a significant cause of delay $(8 \%)$. Because causes of delay were reported by GPs there was a potential reporting bias, with delays occurring prior to first consultation or in secondary care possibly being under-reported.

Conclusion Diagnostic delay for patients with upper and lower GI cancers is multi-faceted, with GP appraisal and type of referral perceived as substantial contributors. Interventions aimed at reducing the time to diagnosis should be targeted at the key causes and settings of delay for different cancer sites.

Disclosure of Interest None Declared

\section{REFERENCE}

Walter, F. Webster, A., Scott, S. \& Emery, J. (2012) 'The Andersen Model of total patient delay: A systematic review of its application in cancer diagnosis.' Journal of Health Services Research and Policy Vol.17, No.2, pp.110-118.

\section{OC-071 COMPARISON OF FAECAL M2-PK AND FIT IN SCREENING FOR COLONIC POLYPS IN AN AVERAGE RISK POPULATION}

doi:10.1136/gutjnl-2013-304907.070

1."R Leen, ' $\mathrm{N}$ Shearer, ${ }^{2} \mathrm{C}$ O'Morain, ${ }^{1} \mathrm{D}$ McNamara. 'Department of Gastroenterology, Tallaght Hospital; '2Department of Gastroenterology, Mount Carmel Hospital, Dublin, Ireland 